\title{
Illustration of the Impedance Behaviour of Extremely Low-Profile Coupled Shorted-Patches Antennas for UHF RFID of People
}

\author{
Milan Svanda and Milan Polivka \\ Department of Electromagnetic Field, Faculty of Electrical Engineering, Czech Technical University in Prague, \\ Technická 2, 16627 Prague 6, Czech Republic
}

Correspondence should be addressed to Milan Svanda; svandm1@fel.cvut.cz

Received 4 July 2013; Revised 30 April 2014; Accepted 2 May 2014; Published 28 May 2014

Academic Editor: Francisco Falcone

Copyright (C) 2014 M. Svanda and M. Polivka. This is an open access article distributed under the Creative Commons Attribution License, which permits unrestricted use, distribution, and reproduction in any medium, provided the original work is properly cited.

The recently introduced coupled shorted-patches technique for the design of extremely low-profile UHF RFID tag antennas is used to illustrate the flexibility of selected feeding methods for tuning the antenna input impedance for the complex values required for matching with typical RFID chips. We present parametric studies of the impedance behaviour of dipole-excited and directly excited antennas designed for radiofrequency identification of people in the European UHF frequency band. Our study can significantly facilitate the design of this class of on-body tag antennas.

\section{Introduction}

Small, low-profile, lightweight, and cheap: these are the most frequently used words in connection with antennas for wearable applications. If we add to this sufficient radiation efficiency and immunity of the electrical parameter against the presence of a human body, we have a specification of the basic requirements for wearable antennas. Unfortunately, a number of these demands conflict with each other. Miniaturization of radiator size is limited by fundamental laws [1-3], while frequency bandwidth and radiation efficiency decline with size reduction.

Immunity of the wearable antenna against the influence of the human body is often dealt with by inserting a thin metallic plane that provides additional screening for a single radiator (e.g., a dipole antenna), or it forms an inherent part of the antenna (e.g., patch-type antennas) [4]. In both cases, however, this type of solution significantly affects the electrical properties of the antenna, as will be discussed below. Another requirement for a wearable antenna for use as a UHF RFID tag antenna is that the inductive input impedance complex conjugate to the chip impedance must have a strong capacitive component [5]. A special radiator arrangement, including various more or less sophisticated feeding techniques, must therefore be used.

The coupled shorted-patches technique that has been presented recently in [6-9] enables the design of extremely low-profile antennas (typically below $0.01 \lambda_{0}$ ). These have much better immunity from the influence of a human body situated in the close vicinity than for the same substrate height standard half- and quarter-wavelength microstrip patch antennas. At the same time, the radiation efficiency of the structure is satisfactory-typically better than $50 \%$. The two ways of excitation also enable the input impedance to be tuned for complex values, which is an essential condition for feeding the antenna in the case of RFID chips.

This paper begins by providing a brief summary of the impedance properties and the radiation efficiency of lowprofile wire and patch antennas operating closely spaced above or inherently above metallic planes. The benefits and drawbacks of wire- and patch-type antennas are outlined and discussed.

Further, we illustrate the impedance flexibility of coupled shorted patches for achieving the required complex 
value using parametric changes of the antenna, excitation dipole, and/or tuning slot geometry. This enables the input impedance to be set for typical complex values of UHF RFID chips, that is, approximately $5 \Omega$ to $50 \Omega$ for the real part and $100 \Omega$ to $200 \Omega$ for the imaginary part.

\section{Benefits and Drawbacks of Wire- and Patch-Type Antennas Closely Spaced above Metallic Planes}

2.1. Wire-Type Antennas. A dipole-type antenna closely spaced above a perfect electric conductor (PEC) may be treated as dipole pair with opposite currents using the mirror principle. As mentioned above, the immunity against negative objects is significantly improved if the primary radiator is placed at a relative distance of approximately $\lambda_{g} / 4$ above the PEC plane. In this case, the direct and reflected waves interfere constructively, and in an ideal case the antenna gain may be improved on as far as $3 \mathrm{~dB}$.

However, destructive interferences appear if the distance of radiator $h$ from the plane is a very small fraction of the wavelength. The image currents $\left(I_{2}\right)$ exhibit a direction opposite to the direction of the primary dipole currents $\left(I_{1}\right)$.

The dipole pair input impedance $Z_{\text {in }}$ is then expressed as $Z_{\text {in }}=Z_{11}+Z_{12} I_{1} / I_{2}$, where, for a close metallic plane mirror, the current is $I_{2} \approx I_{1} . Z_{11}$ stands for the self-impedance of the single dipole, while $Z_{12}$ represents the mutual impedance of the dipole and its image [10]. For $h / \lambda \rightarrow 0: Z_{11} \sim$ $Z_{12}$, therefore $Z_{\text {in }} \rightarrow 0$; consequently the dominant part of the input resistance, radiation resistance $R_{\mathrm{rad}} \rightarrow 0$. As a result, both the radiation and the antenna efficiencies decrease significantly. A detailed analysis can be found in [4].

It is simple to apply a distance dielectric pad between the wire radiator (e.g., a planar dipole) and the metallic plane, but this is not a sufficient improvement of the problem with the decrease in radiation efficiency, which was discussed above. Unfortunately, the pad needs to be relatively thick $\left(0.01-0.1 \lambda_{0}\right)$; see [11-13].

Unlike when a perfect electric conductor (PEC) is used, a perfect magnetic conductor (PMC) reflects the electromagnetic wave without a phase shift; that is, the image current $\left(I_{2}\right)$ exhibits a direction that is the same as the direction of the primary dipole currents $\left(I_{1}\right)$. This phenomenon theoretically enables us to place the primary radiator at an arbitrary distance from the shielding PMC plane, without a rise in the destructive interferences.

Artificial magnetic conductors (AMC) or high impedance surfaces (HIS), usually based on mushroom structures as a particular realization of PMC planes, are sometimes considered for use for low-profile antennas. However, narrow frequency bandwidth and complexity of the structure are a major limitation for the use of AMC surfaces [14-18] in the UHF band.

These limitations can be explained on the basis of the parallel resonant circuit model of the cell of a mushroom structure with the resonant frequency, which can be reduced by a rise in the capacity or the inductance values. A rise in capacity can be achieved either by reducing the gap between

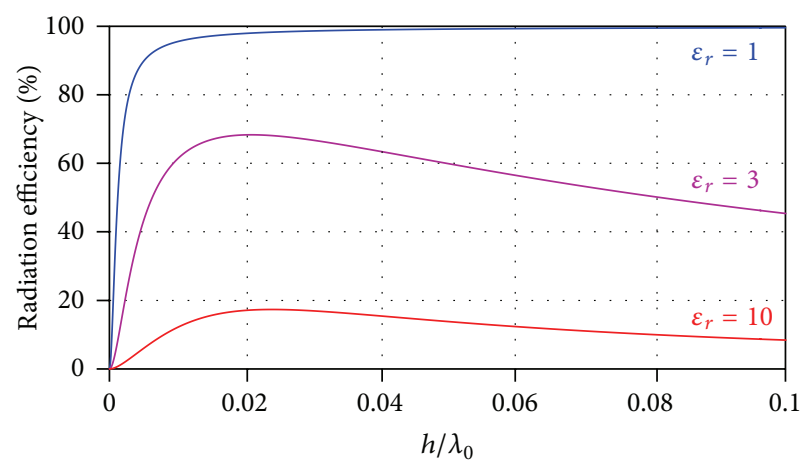

FIGURE 1: Radiation efficiency of a rectangular patch antenna as a function of the relative substrate height; see [27].

the metallic patches or by increasing the patch size. Another way is to increase the shunt inductance via an increase in substrate height. Increasing the capacity has a negative influence on the bandwidth, according to the relation. Increasing the inductance, on the other hand, sets high requirements on the complexity of the structure. Unfortunately, due to the necessity to manufacture shunt inductive vias several millimeters in length in the UHF band [19], their implementation, to date, does not allow us to construct antennas with sufficiently low profiles $\left(h / \lambda_{0}<0.01\right)[4]$. A number of modifications and a property analysis of AMC surfaces have been published, for example, [15, 16, 20-23]. However, they have similar or, indeed, worse properties in the UHF frequency band. Thus, AMC surfaces can be used successfully for improving the performance of the antennas in the high $\mathrm{rf}$ and microwave frequency bands, above approximately $2 \mathrm{GHz}$.

2.2. Patch and PIFA Antennas. The application of antennas based on a metallic ground plane, that is, patch and PIFA antennas [24-26], which can prevent undesirable effects of a nearby object in the proximity of the antenna, is a different approach to these problems. However, at relatively low operational frequencies (hundreds of $\mathrm{MHz}$ ), several potential difficulties must be taken seriously into account. First, when the substrate is less than approximately $0.01 \lambda_{0}$ in height, and the relative permittivity $\varepsilon_{r}$ is higher than that of the air or foam substrate, see Figure 1, their radiation efficiency decreases significantly [27]. Second, the basic patch resonant frequency corresponds to $\lambda_{g} / 2$ or $\lambda_{g} / 4$ and, therefore, at UHF frequencies, patch or PIFA antennas may not be small enough for the intended application.

2.3. Coupled Shorted-Patches Technique. As mentioned in Section 1, the employment of coupled shorted patches enables the design of extremely low-profile antennas, lower than $0.01 \lambda_{0}$, with very good immunity from the influence of a human body located in the close vicinity. The structure is derived from the standard shorted-patches antenna. Despite its many virtues, the standard shorted-patches antenna suffers from a significant fall in its radiation efficiency (below $50 \%)$ if the relative thickness of the substrate drops below approximately $0.01 \lambda_{0}$; see [27]. This phenomenon can be 


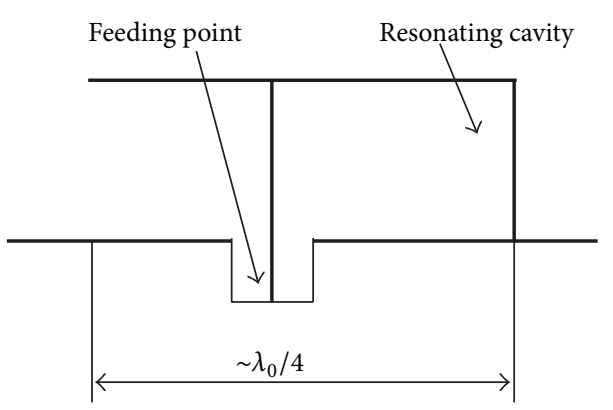

(a)

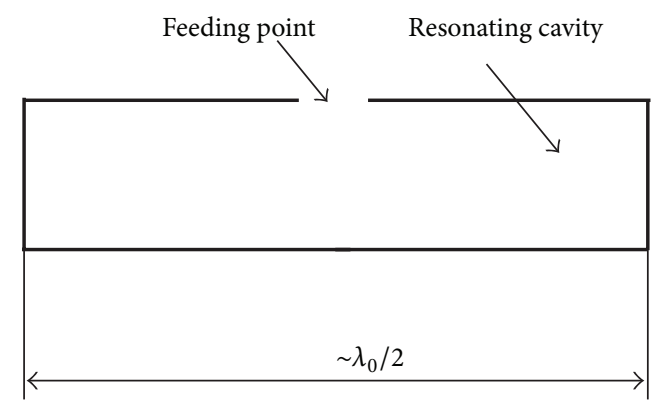

(b)

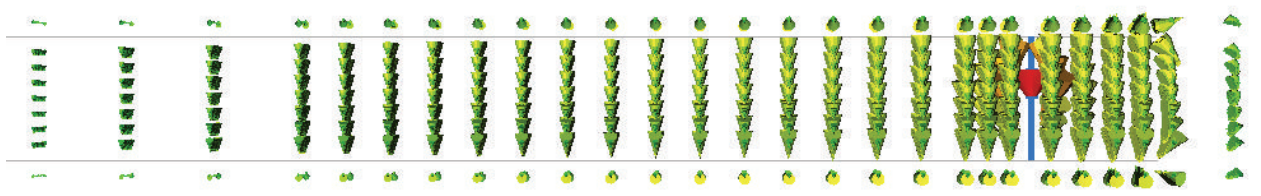

(c)

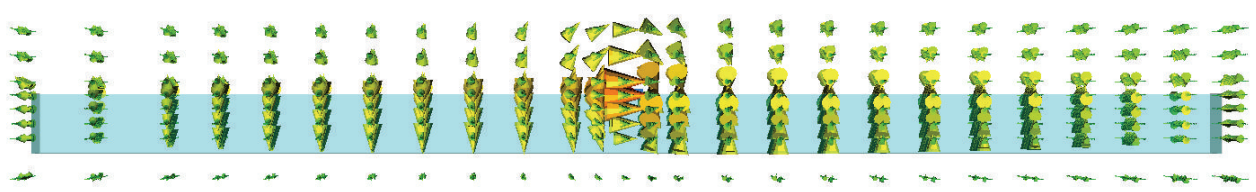

(d)

Figure 2: Cross section ((a) and (b)) and electric field distribution ((c) and (d)) of the standard shorted patch and coupled shorted patches, respectively.

eliminated if there are two quarter-wavelength patches that are strongly coupled by a narrow gap; see Figure 2(b).

The radiation properties of this coupled structure are significantly enhanced even in the case of low-profile substrates with thicknesses below $0.01 \lambda_{0}$ and are, to a large extent, insensitive to the width of the coupling gap. The electric field distributions of the standard shorted patch and coupled shorted patches are demonstrated in Figure 2.

Considering the analogy of the coupled shorted-patches radiator with the common patch antenna, the radiation might be explained on the basis of the transmission line model (TLM) [28]. The radiation of the common rectangular patch antenna is attributed to the electromagnetic fields on the opposite edges along the resonance length of the patch. The phase shift of the normal components of the field to the ground plane is $180^{\circ}$ and, consequently, the far field from these components is minimized. By contrast, the phase shift of the tangential components of the field is $0^{\circ}$ and consequently the far field from these components is maximized. However, the normal E-field component passing through the substrate causes additional dielectric losses. On the other hand, coupled shorted patches concentrate the electric field into the coupling slot going partially through the air above the substrate, and they thus minimize the dielectric losses in the only one coupled radiation slot.

Figure 3 compares the simulated radiation efficiencies of both the standard half-lambda and quarter-lambda patch and coupled shorted-patches antennas, which use the same substrate and have the same footprint size $(60 \times 100 \times 0.76 \mathrm{~mm})$.

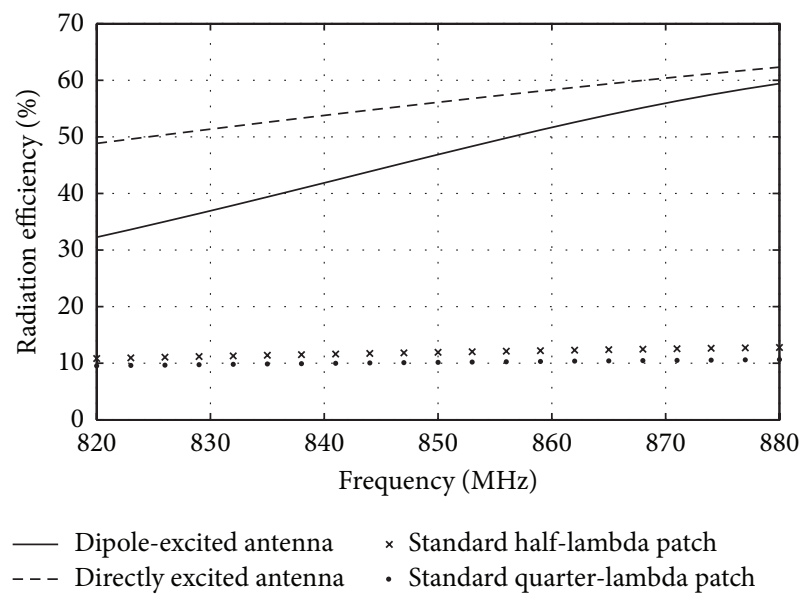

FIGURE 3: A comparison of the radiation efficiency of standard patch and coupled shorted-patches antennas using the same substrate and footprint size $(60 \times 100 \times 0.76 \mathrm{~mm})$.

Standard patches exhibit very low radiation efficiency of about $10 \%$ for $h / \lambda \sim 0.005$, while coupled shorted patches exhibit efficiency better than $50 \%$ in the European frequency band $(865-869 \mathrm{MHz})$.

The coupled shorted-patches antenna can be excited by a linear planar radiator etched on a very thin superstrate, or directly by a symmetrical feeder (e.g., an RFID chip), inserted into the coupling slot between the inner patch edges. Ways of 


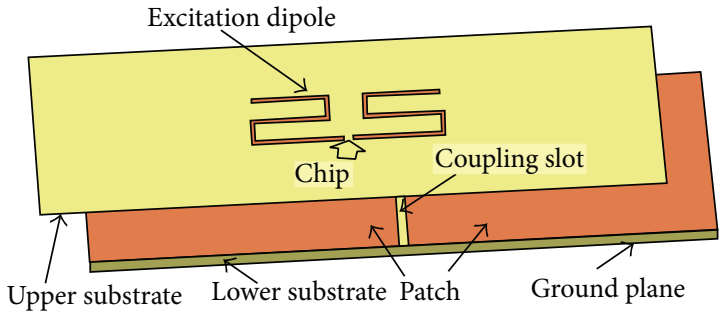

(a)

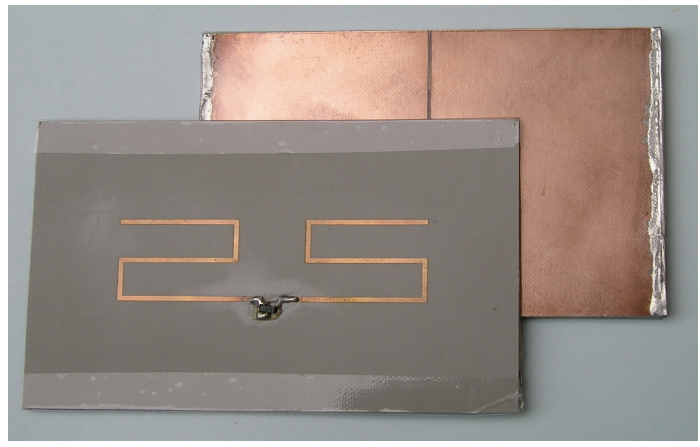

(b)

FIGURE 4: A sketch and a photograph of a dipole-excited coupled shorted-patches antenna.

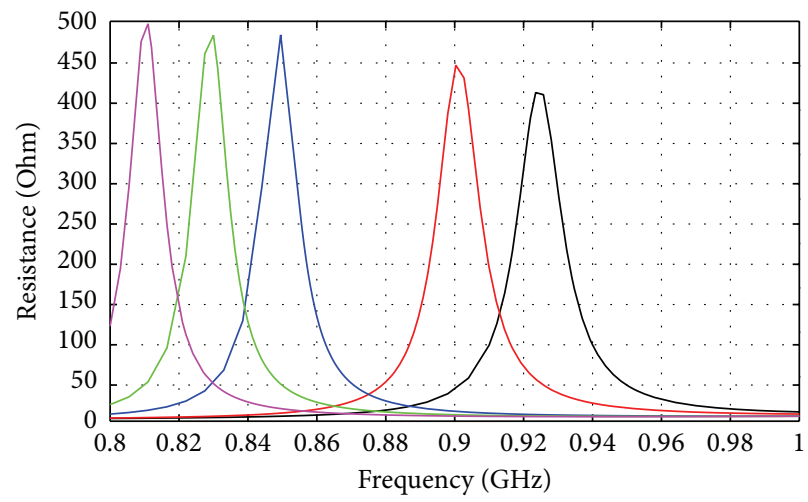

- Patches length $=91.7 \mathrm{~mm}$

- Patches length $=94.1 \mathrm{~mm}$

_ Patches length $=96.5 \mathrm{~mm}$

(a)

Patches length $=98.9 \mathrm{~mm}$

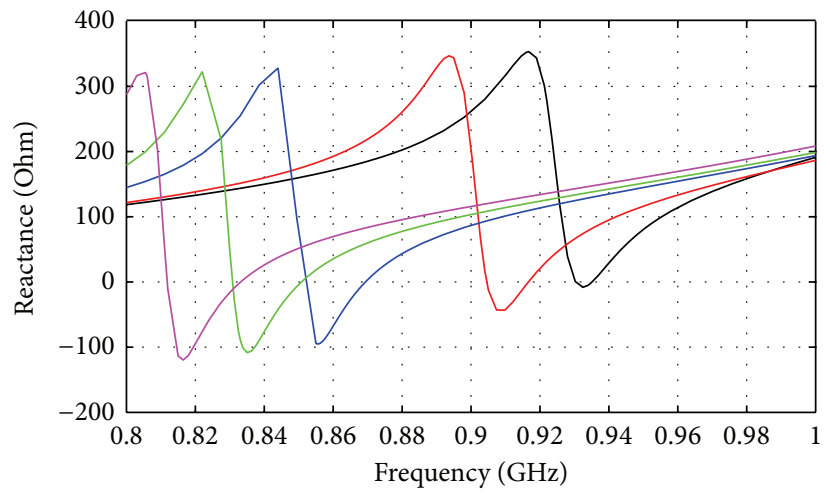
— Patches length $=101.3 \mathrm{~mm}$

- Patches length $=91.7 \mathrm{~mm}$
— Patches length $=94.1 \mathrm{~mm}$
Patches length $=96.5 \mathrm{~mm}$

—_ Patches length $=98.9 \mathrm{~mm}$

- Patches length $=101.3 \mathrm{~mm}$

(b)

FIGURE 5: Tuning the real and imaginary parts of the input impedance by varying the patch length.
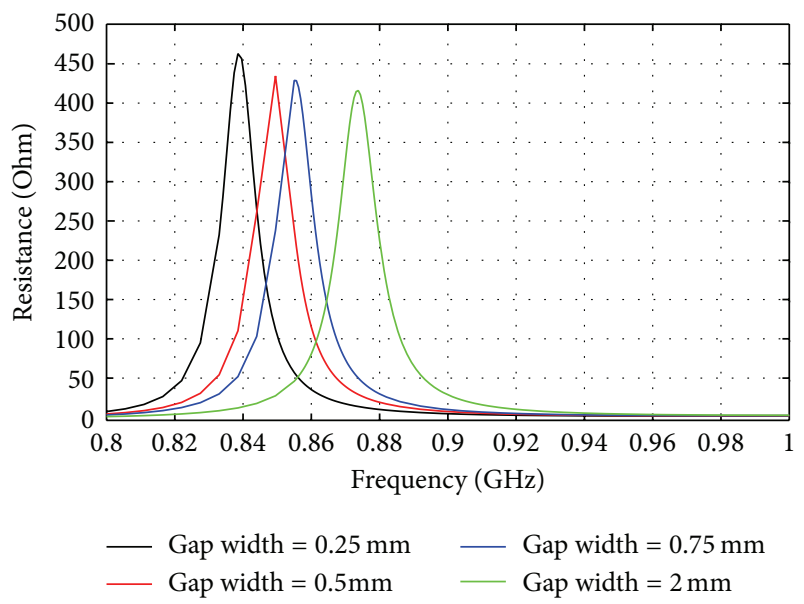

(a)

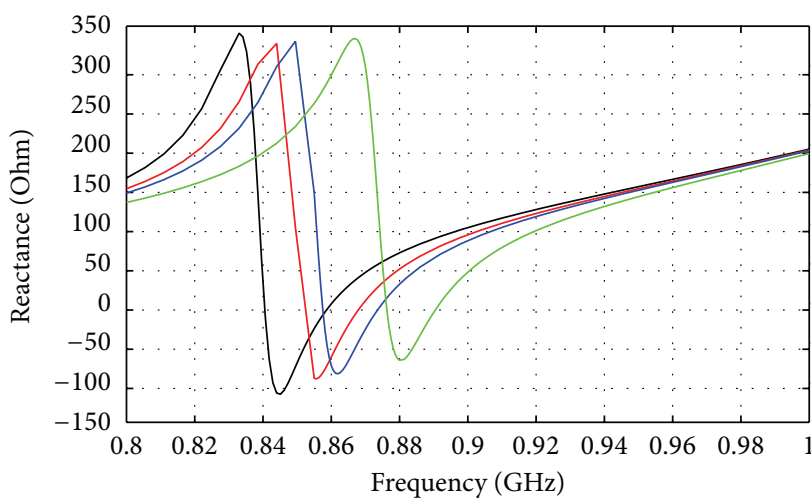

— Gap width $=0.25 \mathrm{~mm} \quad$ Gap width $=0.75 \mathrm{~mm}$

- Gap width $=0.5 \mathrm{~mm} \quad$ Gap width $=2 \mathrm{~mm}$

(b)

FIGURE 6: Tuning the real and imaginary parts of the input impedance by varying the width of the coupling gap. 


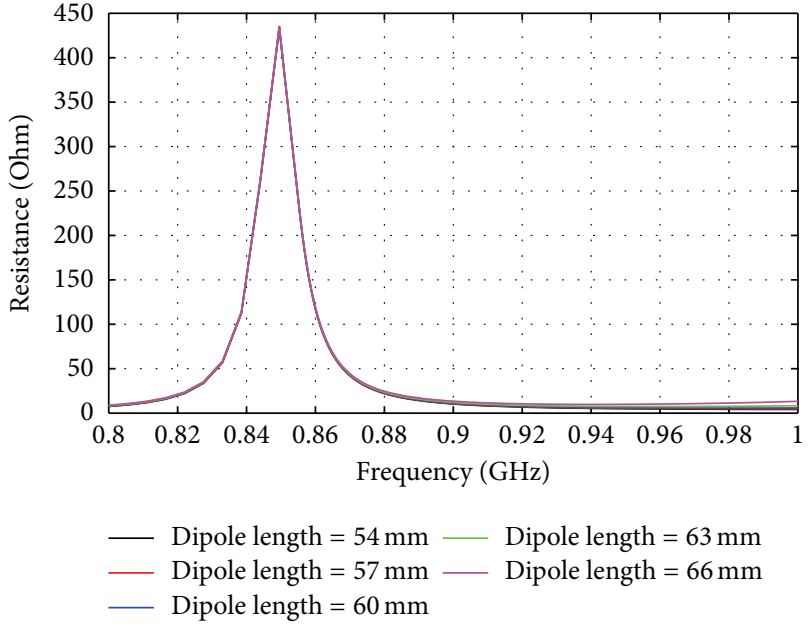

(a)

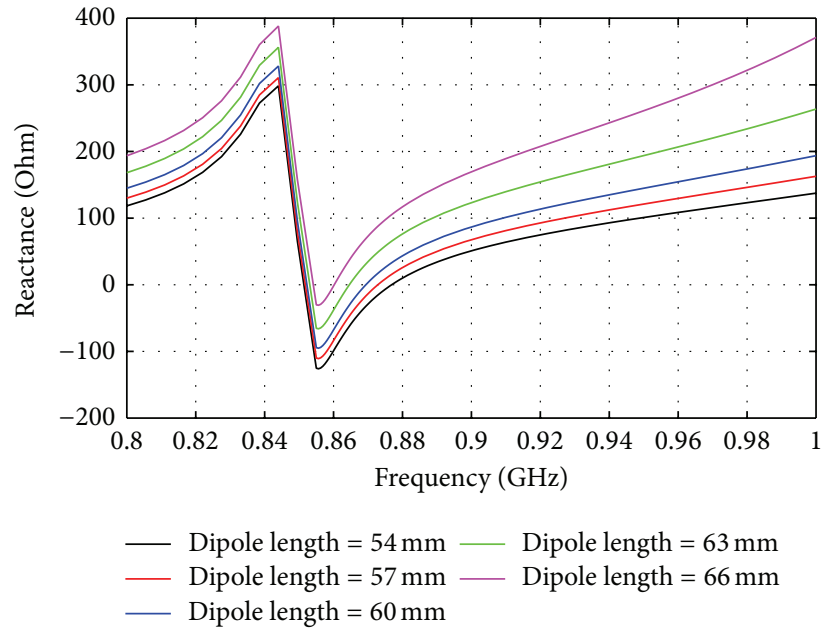

(b)

FIGURE 7: Tuning the real and imaginary parts of the input impedance by varying the length of the excitation dipole.

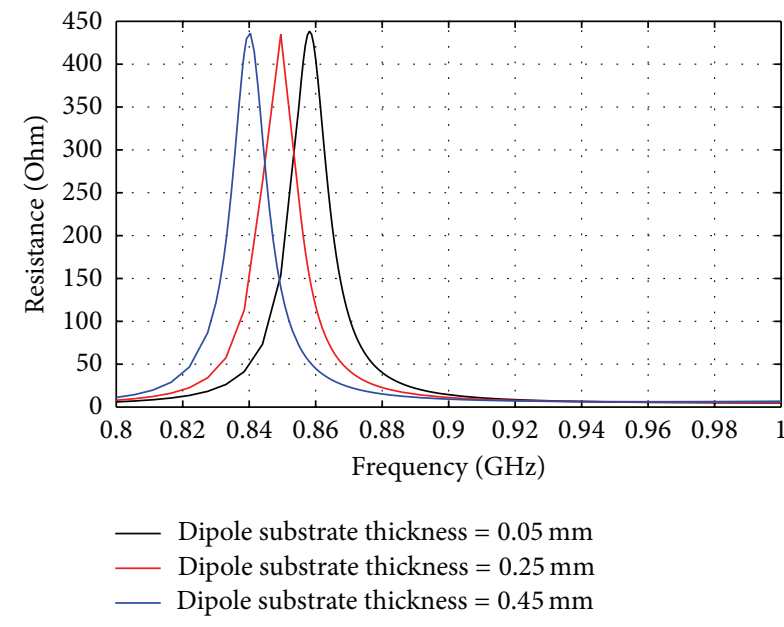

(a)

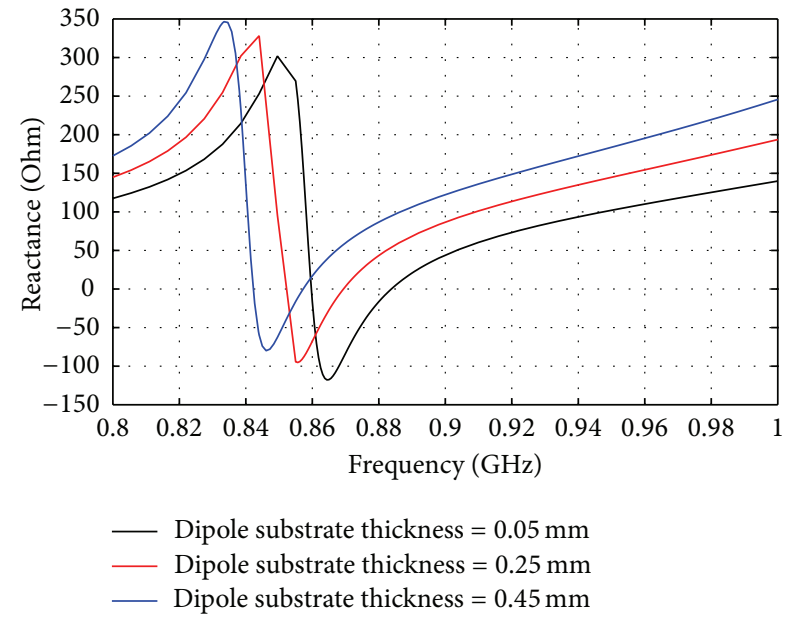

(b)

FIGURE 8: Tuning the real and imaginary parts of the input impedance by varying the height of the superstrate layer.

tuning and matching the impedance to the chip impedance using the two types of feeding are described in the following section.

\section{Parametric Study of Tuning the Input Impedance of Coupled Shorted Patches}

3.1. Dipole-Excited Coupled Shorted Patches. The total size of the dipole-excited coupled shorted-patches antenna considered here is $60 \times 95 \times 1 \mathrm{~mm}$ (the relative size is equal to $0.17 \times 0.28 \times 0.003 \lambda_{0}$ at $869 \mathrm{MHz}$ ). The dielectric constant accounts for $\varepsilon_{r}=3.2$, while its loss tangent reaches tan $\delta=0.002$. A planar double meander folded dipole $1 \mathrm{~mm}$ in width (see Figure 4) and $58 \times 16 \mathrm{~mm}$ in outer size is used as an excitation element. However, other radiator shapes might be considered, such as a meander dipole, a folded dipole, and a loop antenna; see $[6,29-31]$.
The principle for setting the antenna impedance for the required complex value at the specified frequency of the European UHF RFID band is based on the change in the current distribution on the excitation meander dipole by modifying its geometry and size in conjunction with modifying the size of the coupled-patches structure. The parametric study employs impedance-sensitive dimensions such as patch length, coupling gap width, excitation dipole length, and superstrate height to tune the input impedance for a complex conjugate value to the RFID chip impedance $Z_{\text {chip }}=76-j 340 \Omega$ at observation frequency $869 \mathrm{MHz}$. The changes are applied just to this one parameter, while the other parameters remain the same as mentioned above.

The first study presents the sensitivity of the antenna impedance to the patch length; see Figure 5. The real part varies between a few and $400 \Omega$, and the imaginary part varies 


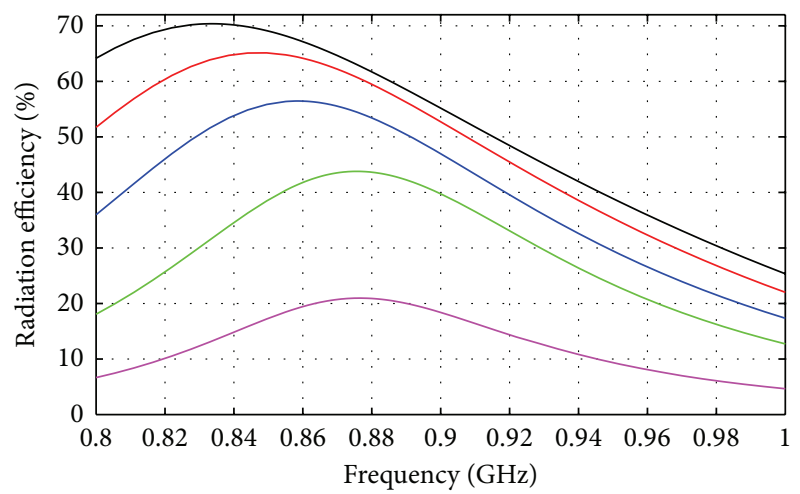

Substrate thickness $=1.22 \mathrm{~mm}-$ Substrate thickness $=0.53 \mathrm{~mm}$
Substrate thickness $=0.99 \mathrm{~mm}-$ Substrate thickness $=0.3 \mathrm{~mm}$
Substrate thickness $=0.76 \mathrm{~mm}$

FIGURE 9: Radiation efficiency as a function of patch substrate height.

between $-100 \Omega$ and $350 \Omega$ at observation frequency for a patch size between 94 and $96.5 \mathrm{~mm}$.

Significant sensitivity of the input impedance depends on the width of the coupling gap, where changes between 0.75 and $2.0 \mathrm{~mm}$ cause a variation of the input resistance between $50 \Omega$ and $420 \Omega$ and input reactance between $-100 \Omega$ and $320 \Omega$; see Figure 6.

Further, the length of an excitation dipole placed on a superstrate layer effectively changes just the input reactance between $-50 \Omega$ and $50 \Omega$ for dipole length varying between 54 and $66 \mathrm{~mm}$; see Figure 7.

Finally, varying the height of the superstrate layer between 0.05 and $0.45 \mathrm{~mm}$ changes the input resistance between approximately 25 and $100 \Omega$ and changes the reactance between $-60 \Omega$ and $60 \Omega$, respectively; see Figure 8 .

As can be seen from the previous study, basic tuning of the antenna input impedance is performed by changing the patch length, whereby the radiation efficiency in the vicinity of the maximum is ensured simultaneously. The patch length should be set to a quarter-wavelength on the substrate that is used. Then finer tuning can be achieved by means of the dipole length and the gap width.

The radiation efficiency is dominantly affected by the height of the patch substrate; see Figure 9. The other tracked parameters do not have a significant influence.

\subsection{Directly Excited Coupled Shorted Patches with Tuning} Slots. Another way to simplify the structure of the radiator is by removing the upper substrate and exciting directly by the symmetrical feeder, inserted into the slot situated between the inner patch edges. Unfortunately, this structure does not suffice the capability of the impedance tuning. However, this could be solved by inserting two tuning slots as reactive elements placed symmetrically on both sides of the coupling slot; see Figure 10.

The total size of the directly excited coupled-patches antenna is $60 \times 100 \times 0.76 \mathrm{~mm}$ (the relative size is $0.17 \times 0.29 \times$ $0.0022 \lambda_{0}$ at $869 \mathrm{MHz}$ ). The dimensions of the impedance tuning slots are as follows: length $40 \mathrm{~mm}$, width $6 \mathrm{~mm}$, and distance from the coupling gap $7 \mathrm{~mm}$. The antenna is performed on a low-permittivity substrate $\varepsilon_{r}=3.2$, while its loss tangent is $\tan \delta=0.002$.

The principle for setting the antenna impedance for the required complex values at the specified frequency is based on the change in the field distribution of the inner part of the coupled patches using the tuning slots and consequently the overall antenna size. The parametric study again employs impedance-sensitive dimensions such as patch length and width, coupling gap width, tuning slot length, and their distance from the coupling gap to tune the input impedance for the complex conjugate value to the RFID chip impedance $Z_{\text {chip }}=76-j 340 \Omega$ at observation frequency $869 \mathrm{MHz}$. The changes are again applied just to the one parameter, while the other parameters remain the same, as mentioned above.

The first study of a directly fed shorted-patches antenna presents the sensitivity of the antenna impedance to the patch length and width; see Figure 11. The input resistance varies between a few and approximately $50 \Omega$, and the imaginary part varies between $90 \Omega$ and $230 \Omega$ at observation frequency for a patch length between 95 and $105 \mathrm{~mm}$. This extent is not sufficient for matching the intended RFID chip. In addition, the input resistance varies between approximately 20 and $1100 \Omega$, and the imaginary part varies between 200 and $500 \Omega$ at observation frequency for a patch width between 42 and $78 \mathrm{~mm}$.

There is significant sensitivity of the input impedance to the width of the coupling gap, where changes between 0.3 and $5.7 \mathrm{~mm}$ cause variation of the input resistance between a few $\Omega$ and $600 \Omega$ and variation in input reactance between 120 and $400 \Omega$; see Figure 12 .

Further, the length of the tuning slots effectively changes both the input resistance between a few and $600 \Omega$ and the reactance between $-280 \Omega$ and $380 \Omega$ for length varying from 160 to $168 \mathrm{~mm}$; see Figure 13.

Finally, varying the distance of the tuning slot from a coupling slot between 1.5 and $4.5 \mathrm{~mm}$ changes the input resistance between a few and $120 \Omega$ and changes the reactance between $140 \Omega$ and $340 \Omega$; see Figure 14 .

Similarly, as for the dipole-excited case, basic tuning of the antenna input impedance can be performed by changing the patch length, whereby the radiation efficiency in the vicinity of the maximum is ensured simultaneously. The patch length should be closer to the quarter-wavelength on the substrate that is used. Then finer tuning can be achieved by means of the gap width and by tuning the position and the width of the slots.

The radiation efficiency is again dominantly affected by the height of the patch substrate; see Figure 15. The other tracked parameters have no significant influence.

\section{Measurement and Identification Tests}

Both antenna prototypes discussed above were manufactured and their performance properties were verified by measurement. The simulated and measured reflection coefficient, the total efficiency in free space and in the 


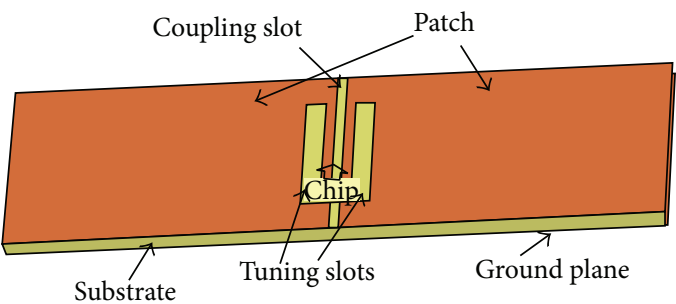

(a)

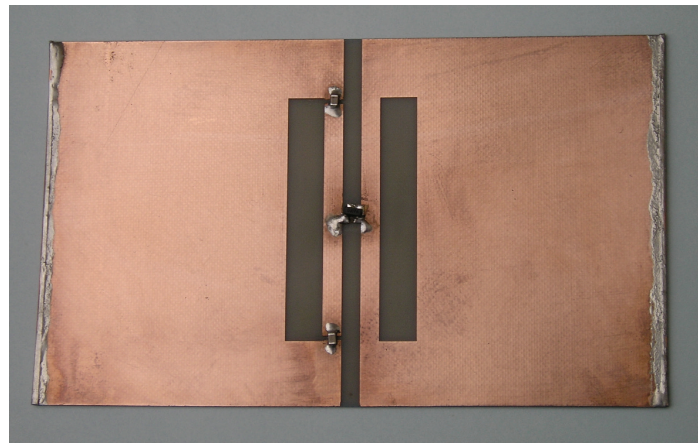

(b)

FiguRE 10: Sketch and photograph of the directly excited coupled shorted-patches antenna with tuning slots.

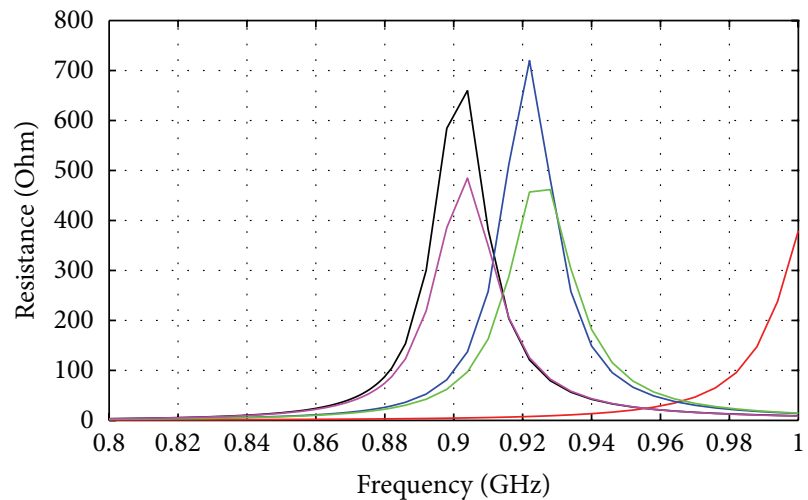

— Patches length $=95 \mathrm{~mm}$

Patches length $=97.5 \mathrm{~mm}$

- Patches length $=100 \mathrm{~mm}$

(a)

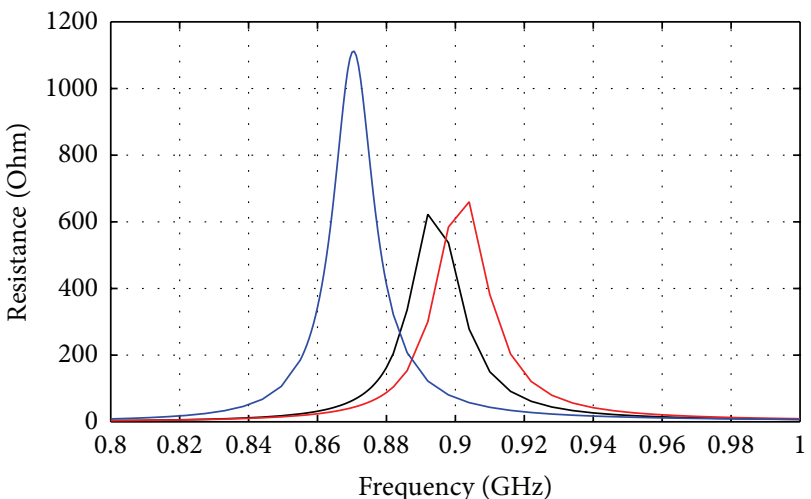

Patch width $=42 \mathrm{~mm}$
Patch width $=60 \mathrm{~mm}$
Patch width $=78 \mathrm{~mm}$

(c)

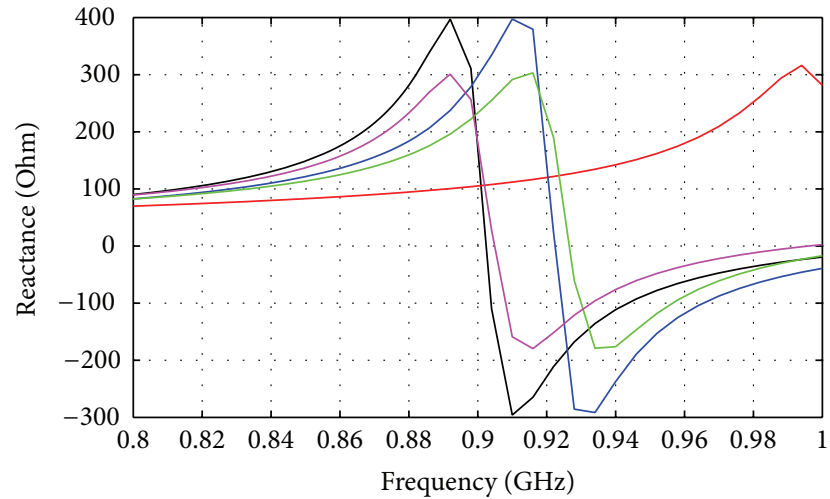
- Patches length $=95 \mathrm{~mm}$
Patches length $=97.5 \mathrm{~mm}$
- Patches length $=102.5 \mathrm{~mm}$
_ Patches length $=105 \mathrm{~mm}$
- Patches length $=100 \mathrm{~mm}$

(b)

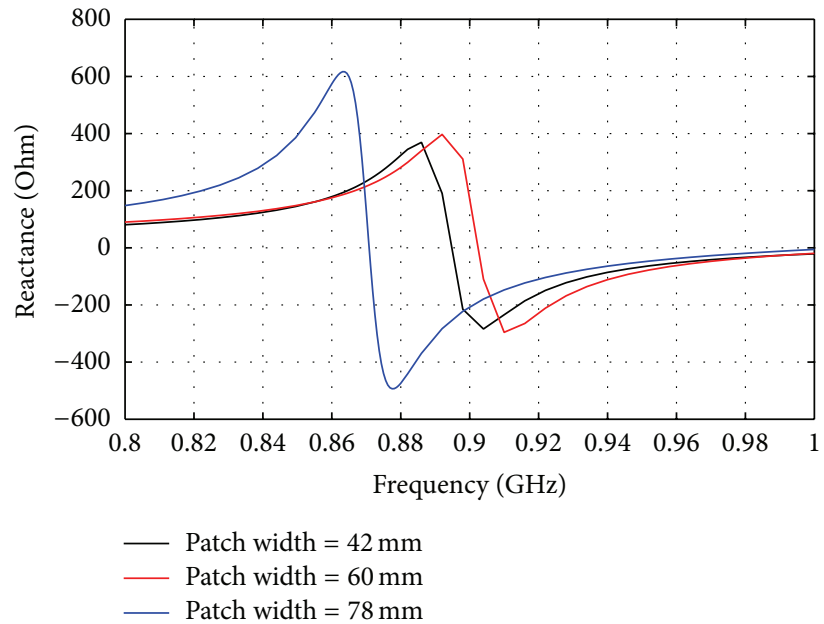

(d)

FIGURE 11: Tuning the (a) real and (b) imaginary parts of the input impedance by varying the patch length, and the same ((c) and (d)) for patch width. 

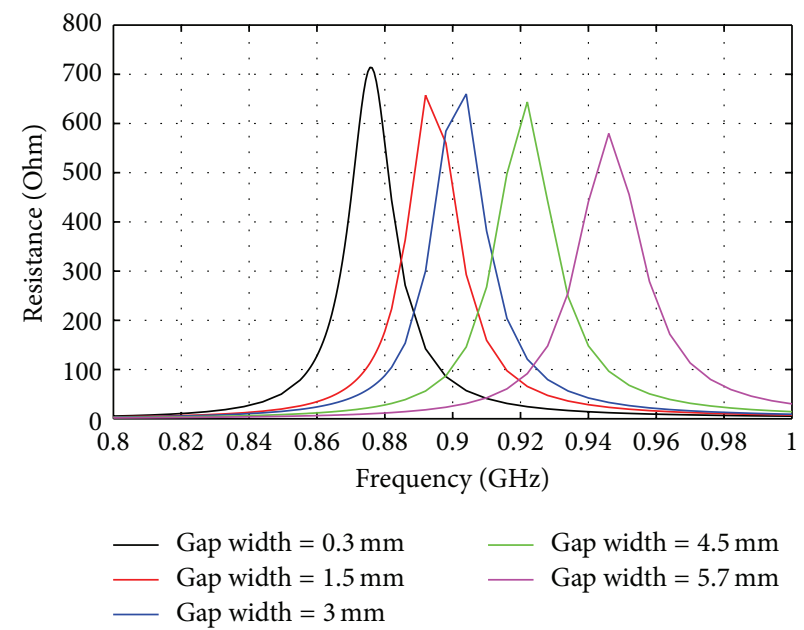

(a)

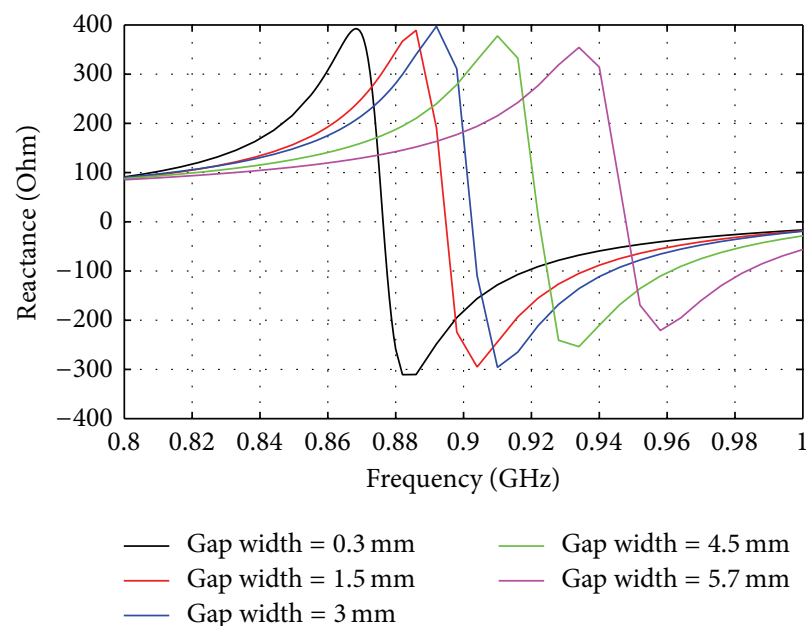

(b)

FIGURE 12: Tuning the real and imaginary parts of the input impedance by varying the width of the coupling gap.

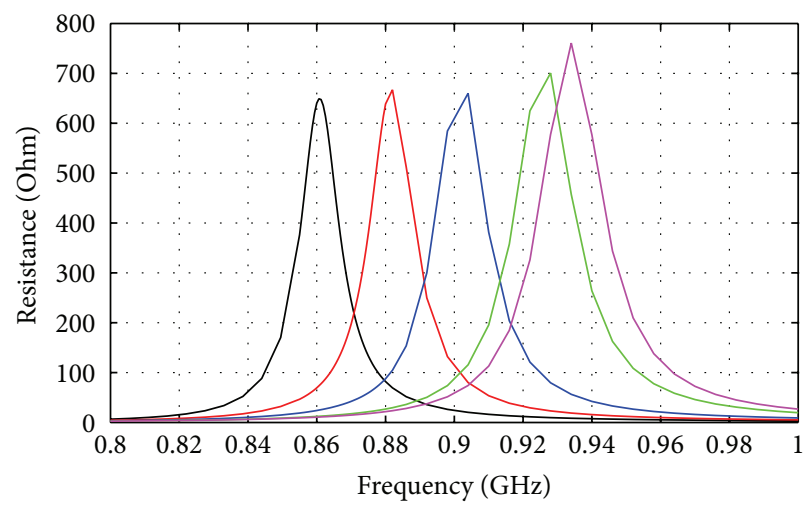

Turning slot length $=160 \mathrm{~mm} \quad-\quad$ Turning slot length $=164.8 \mathrm{~mm}$
Turning slot length $=163.2 \mathrm{~mm} \quad \begin{aligned} & \text { Turning slot length }=168.1 \mathrm{~mm} \\ & \text { Turning slot length }=164 \mathrm{~mm}\end{aligned} \quad$.

(a)
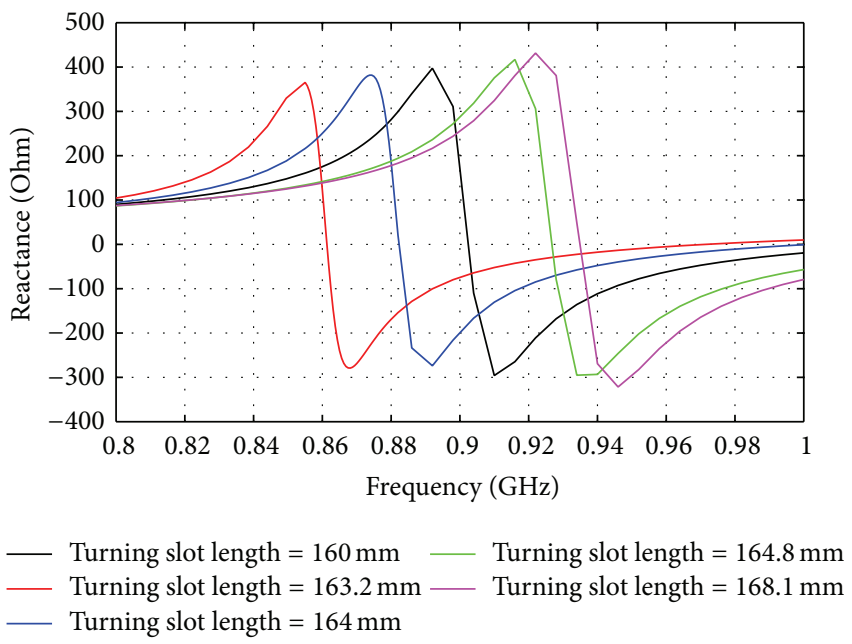

(b)

FIGURE 13: Tuning the real and imaginary parts of the input impedance by varying the tuning slot length.

TABLE 1: Simulated and measured electrical parameters of coupled-patch antennas.

\begin{tabular}{|c|c|c|c|c|c|c|}
\hline Antenna & $S_{11, \text { free }}(\mathrm{dB})$ & $S_{11, \text { body }}(\mathrm{dB})$ & $\eta_{t, \text { free }}(\%)$ & $\eta_{t, \text { body }}(\%)$ & $G_{\text {free }}(\mathrm{dBi})$ & $G_{\text {body }}(\mathrm{dBi})$ \\
\hline Dipole-excited simulation & -28 & -15 & 54 & 57 & 1 & 1.1 \\
\hline Dipole-excited measurement & -13 & -16 & 47 & 64 & 1.3 & 1.7 \\
\hline Directly excited simulation & -26 & -18 & 56 & 58 & 1.1 & 0.9 \\
\hline Directly excited measurement & -23 & -14 & 52 & 55 & 1.0 & 1.6 \\
\hline
\end{tabular}

TABLE 2: Measured maximum identification distance in the corridor of building $4 \mathrm{~m}$ in width and $3.5 \mathrm{~m}$ in height.

\begin{tabular}{lcc}
\hline Tested configuration & Reader and tag antenna axis offset $[\mathrm{m}]$ & Reach of correct identification $d_{\text {max }}[\mathrm{m}]$ \\
\hline Dipole-excited antenna on the chest of a person & 0 & 7.5 \\
\hline Directly excited antenna on the chest of a person & 1.8 & 7 \\
\hline
\end{tabular}




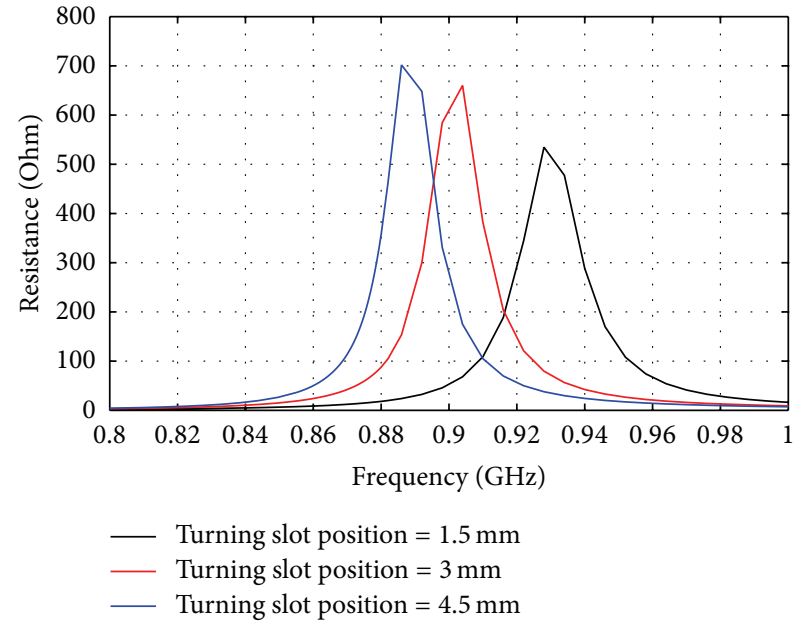

(a)

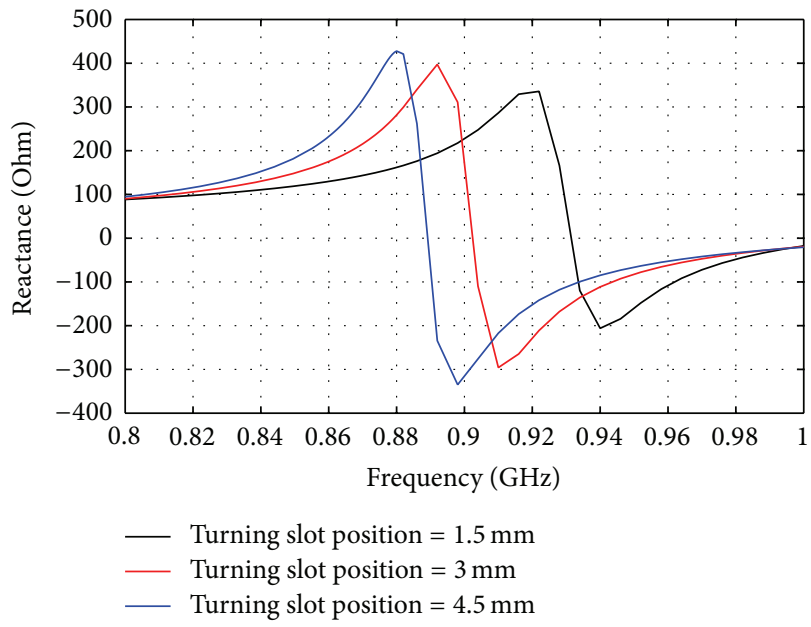

(b)

FIGURE 14: Tuning the real and imaginary parts of the input impedance by changing the distance of the tuning slot from the coupling gap.

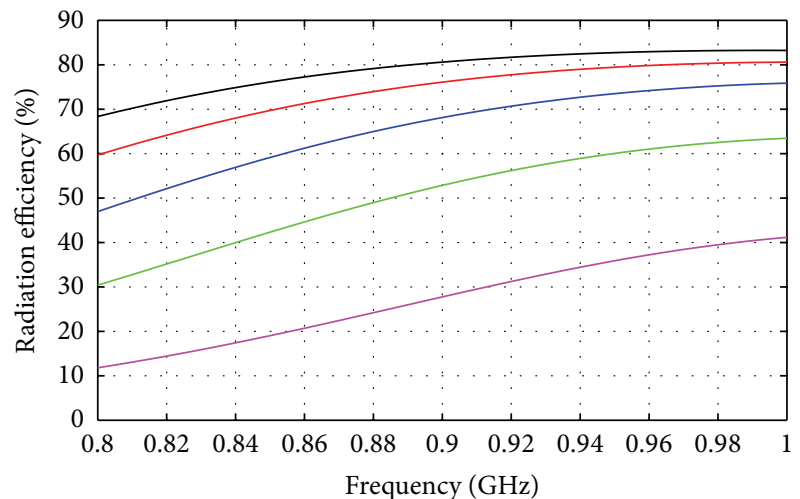

Substrate thickness $=1.22 \mathrm{~mm}-$ Substrate thickness $=0.53 \mathrm{~mm}$
Substrate thickness $=0.99 \mathrm{~mm}-$ Substrate thickness $=0.3 \mathrm{~mm}$
Substrate thickness $=0.76 \mathrm{~mm}$

FIGURE 15: Radiation efficiency of directly fed coupled shorted patches with tuning slots as a function of the height of the patch substrate.

close vicinity of a human body of both coupled shortedpatches antennas and the power gain are compared in Table 1. Good immunity against the influence of a human body can be seen for the final matched prototypes.

The measurement of the designed antenna was carried out in the monopole arrangement, in order to avoid the use of a balun situated between the antenna and the coaxial connector. The monopole input impedance then accounts for a half of the value compared to the dipole impedance [8]. Consequently, $Z_{\text {monopole }}=Z_{\text {dipole }} / 2$ is considered for further evaluation (where $Z_{\text {dipole }}=Z_{\text {chip }}{ }^{*}=76+j 340 \Omega$ ). The radiation efficiency was evaluated from the impedance measurement performed using the Wheeler cap method [32]. The cap size was equal to $122 \times 122 \times 122 \mathrm{~mm}$. The measurement was performed with and without a human body phantom (manufactured from agar with $\varepsilon_{r} \sim 55$ and tan $\delta \sim 0.5$ of $80 \times 110 \times 15 \mathrm{~mm}$ size), which was enclosed into the back of the antenna.

In order to evaluate the performance of the tag antennas in real system conditions, a read range test was performed in a $4 \mathrm{~m}$ wide building corridor. Tag antennas with the chip were fixed at a height of $1.25 \mathrm{~m}$ on the chest of a person. The test results are included in Table 2.

\section{Conclusion}

An illustration has been provided of the flexibility of the tuning of coupled shorted-patches RFID tag antennas for the required complex input impedance using two different feeding techniques. The parametric studies of the influence of geometrical modifications on the input impedance behaviour can significantly facilitate the design of this class of on-body tag antennas.

The prototypes presented here were matched to RFID chip impedance $Z_{\text {chip }}=76+j 340 \Omega$ at frequency $869 \mathrm{MHz}$ with concurrent very good immunity from the influence of a human body situated in the direct vicinity of the antenna. The measured radiation efficiency of the structure was sufficient, typically better than $50 \%$.

\section{Conflict of Interests}

The authors declare that there is no conflict of interests regarding the publication of this paper.

\section{Acknowledgments}

This research was undertaken at the Department of Electromagnetic Field at the Czech Technical University in Prague. It was supported by the Czech Science Foundation Project no. P102/12/P863 "Electromagnetic Properties of Radiating Structures and Artificial Screening Surfaces in the Close Vicinity of the Human Body." 


\section{References}

[1] H. A. Wheeler, "Fundamental limitations in antennas," Proceedings of the IEEE, vol. 35, pp. 1479-1484, 1947.

[2] R. E. Collin and S. Rothschild, "Evaluation of antenna Q," IEEE Transaction on Antennas and Propagation, vol. 12, no. 1, pp. 2327, 1964.

[3] L. J. Chu, "Physical limitations of omni-directional antennas," Journal of Applied Physics, vol. 19, no. 12, pp. 1163-1175, 1948.

[4] M. Polivka, M. Svanda, and P. Hudec, "UHF RFID of people," in Development and Implementation of RFID Technology, C. Turcu, Ed., chapter 4, pp. 63-88, In-Tech, Vienna, Austria, 2009.

[5] K. Finkenzeller, RFID Handbook: Fundamentals and Applications in Contactless Smart Cards and Identification, John Wiley \& Sons, New York, NY, USA.

[6] M. Polivka, M. Svanda, P. Hudec, and S. Zvanovec, "UHF RF identification of people in indoor and open areas," IEEE Transactions on Microwave Theory and Techniques, vol. 57, no. 5, pp. 1341-1347, 2009.

[7] M. Svanda and M. Polivka, "Novel dual-loop antenna placed over patch array surface for UHF RFID of dielectric and metallic objects," Microwave and Optical Technology Letters, vol. 51, no. 3, pp. 709-713, 2009.

[8] M. Švanda and M. Polívka, “Two novel extremely low-profile slot-coupled two-element patch antennas for UHF RFID of people," Microwave and Optical Technology Letters, vol. 52, no. 2, pp. 249-252, 2010.

[9] G. Marrocco, "RFID antennas for the UHF remote monitoring of human subjects," IEEE Transactions on Antennas and Propagation, vol. 55, no. 6, pp. 1862-1870, 2007.

[10] C. A. Balanis, Antenna Theory, Analysis and Design, John Wiley \& Sons, New York, NY, USA, 1997.

[11] G. A. Conway and W. G. Scanlon, "Low-profile patch antennas for over-body-surface communication at $2.45 \mathrm{GHz}$," in Proceedings of the IEEE International Workshop on Antenna Technology (iWAT '07), pp. 416-419, March 2007.

[12] D. C. Ranasinghe, D. M. Hall, P. H. Cole, and D. W. Engels, "An embedded UHF RFID label antenna for tagging metallic objects," in Proceedings of the Intelligent Sensors, Sensor Networks and Information Processing Conference (ISSNIP '04), pp. 343-347, December 2004.

[13] J. Siden, H. E. Nilsson, A. Koptyug, and T. Olsson, "A distanced RFID dipole for a metallic supply chain label," in Proceedings of IEEE Antennas and Propagation Society International Symposium, pp. 3229-3232, 2006.

[14] D. Sievenpiper, L. Zhang, R. F. J. Broas, N. G. Alexöpolous, and E. Yablonovitch, "High-impedance electromagnetic surfaces with a forbidden frequency band," IEEE Transactions on Microwave Theory and Techniques, vol. 47, no. 11, pp. 2059-2074, 1999.

[15] D. F. Sievenpiper, High-impedance electromagnetic surfaces [Ph.D. dissertation], University of California, Los Angeles, Los Angeles, Calif, USA, 1999.

[16] A. P. Feresidis, G. Goussetis, S. Wang, and J. C. Vardaxoglou, "Artificial magnetic conductor surfaces and their application to low-profile high-gain planar antennas," IEEE Transactions on Antennas and Propagation, vol. 53, no. 1, pp. 209-215, 2005.

[17] Y. Zhang, J. von Hagen, M. Younis, C. Fischer, and W. Wiesbeck, "Planar artificial magnetic conductors and patch antennas," IEEE Transactions on Antennas and Propagation, vol. 51, no. 10, pp. 2704-2712, 2003.
[18] S. Tretyakov, Analytical Modeling in Applied Electromagnetics, Artech House, 2003.

[19] Z. Li, G. Wang, and Y. Cao, "A low-profile equiangular spiral antenna using a novel EBG ground plane," in Proceedings of the 7 th International Symposium on Antennas, Propagation and EM Theory (ISAPE '06), pp. 1-3, October 2006.

[20] G. Goussetis, A. P. Feresidis, and J. C. Vardaxoglou, “Tailoring the AMC and EBG characteristics of periodic metallic arrays printed on grounded dielectric substrate," IEEE Transactions on Antennas and Propagation, vol. 54, no. 1, pp. 82-89, 2006.

[21] S. Clavijo, R. E. Díaz, and W. E. McKinzie III, "Design methodology for sievenpiper high-impedance surfaces: an artificial magnetic conductor for positive gain electrically small antennas," IEEE Transactions on Antennas and Propagation, vol. 51, no. 10, pp. 2678-2690, 2003.

[22] M. Hosseini, A. Pirhadi, and M. Hakkak, "A novel AMC with little sensitivity to the angle of incidence using 2-layer jerusalem cross FSS," Progress in Electromagnetics Research, vol. 64, pp. 4351, 2006.

[23] Y. Kim, F. Yang, and A. Z. Elsherbeni, "Compact artificial magnetic conductor designs using planar square spiral geometries," Progress in Electromagnetics Research, vol. 77, pp. 43-54, 2007.

[24] L. Ukkonen, L. Sydänheimo, and M. Kivikoski, "Effects of metallic plate size on the performance of microstrip patch-type tag antennas for passive RFID," IEEE Antennas and Wireless Propagation Letters, vol. 4, no. 1, pp. 410-413, 2005.

[25] L. Ukkonen, D. Engels, L. Sydänheimo, and M. Kivikoski, "Planar wire-type inverted-F RFID tag antenna mountable on metallic objects," in Proceedings of the IEEE Antennas and Propagation Society Symposium, vol. 1, pp. 101-104, June 2004.

[26] M. Hirvonen, P. Pursula, K. Jaakkola, and K. Laukkanen, "Planar inverted-F antenna for radio frequency identification," Electronics Letters, vol. 40, no. 14, pp. 848-850, 2004.

[27] K. F. Lee and W. Chen, Advances in Microstrip and Printed Antennas, John Wiley \& Sons, New York, NY, USA, 1997.

[28] J. R. James and P. S. Hall, Handbook of Microstrip Antennas, Peter Peregrinus, 1989.

[29] M. Svanda and M. Polivka, "Low profile antenna," Czech Republic Patent no. 303264, 2012.

[30] M. Svanda, M. Polivka, and P. Hudec, "Novel low-profile foam dielectric over-the-shoulder antenna based on coupled patches technique," Microwave and Optical Technology Letters, vol. 55, no. 3, pp. 593-597, 2013.

[31] P. Hudec, M. Svanda, and M. Polivka, "Communication antenna intended for fastening on human body," Czech Republic Patent no. 302377, 2011.

[32] H. A. Wheeler, "The radian sphere around a small antenna," Proceedings of the IRE, vol. 47, no. 8, pp. 1325-1331, 1959. 

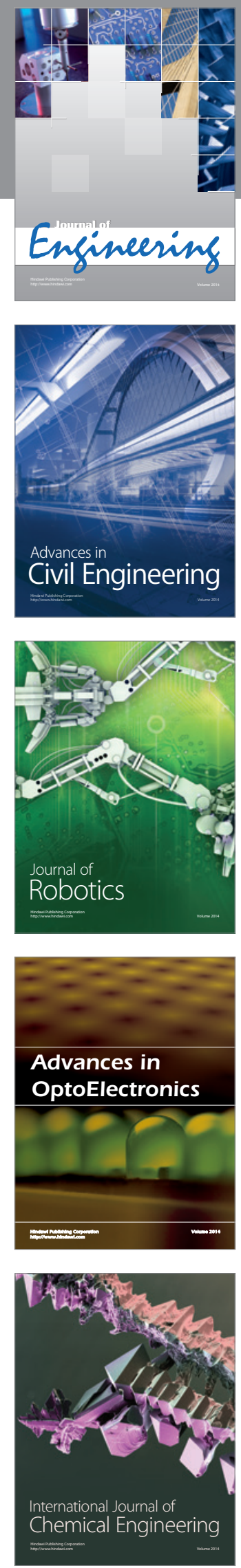

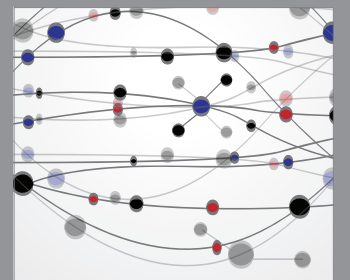

The Scientific World Journal
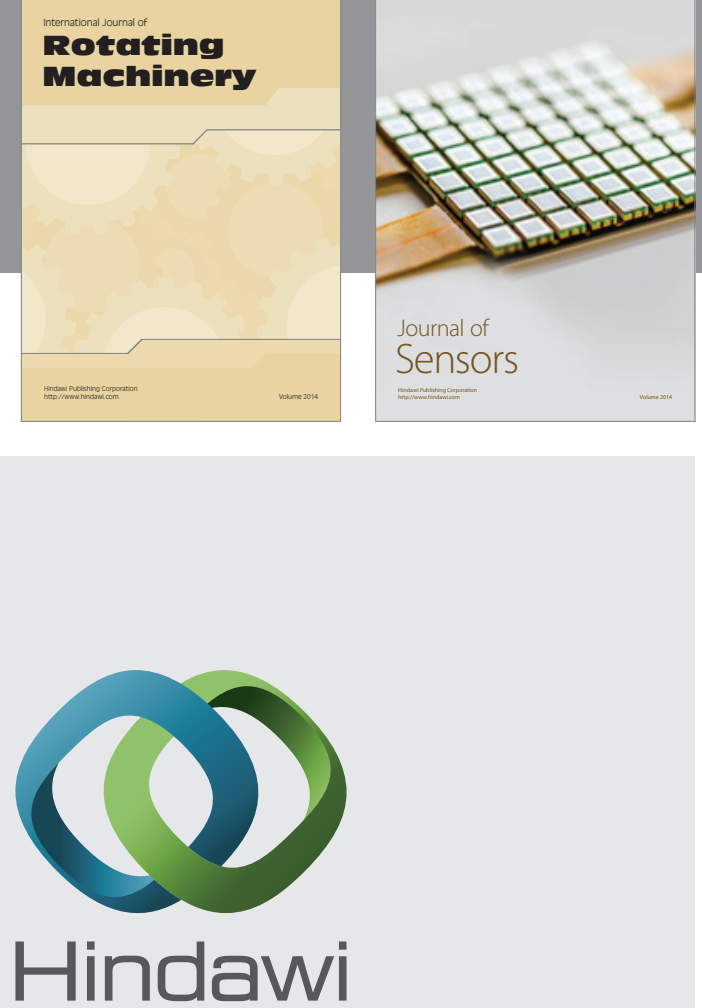

Submit your manuscripts at http://www.hindawi.com
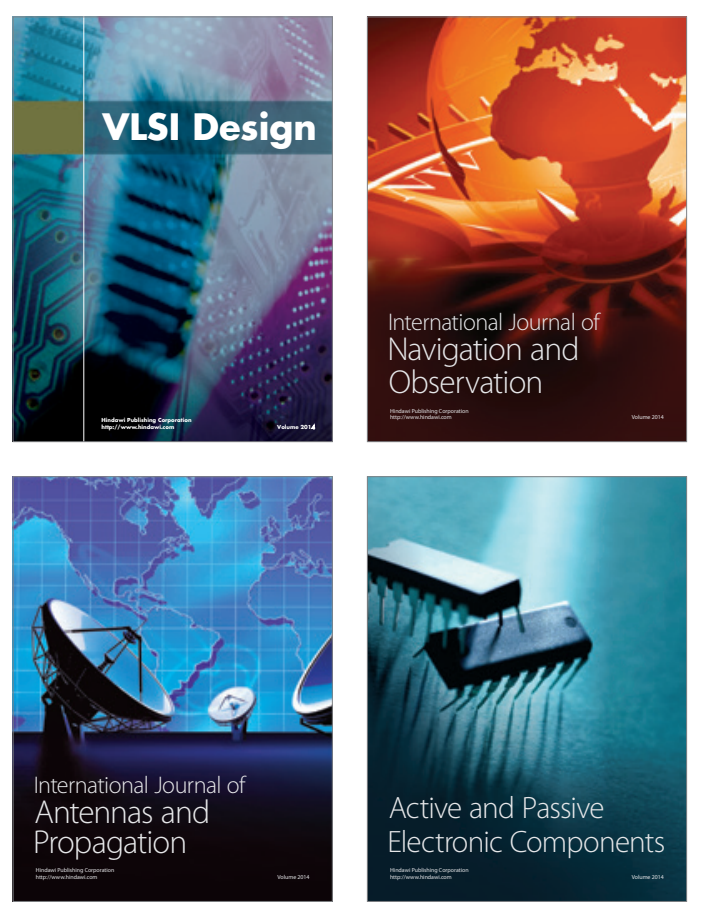
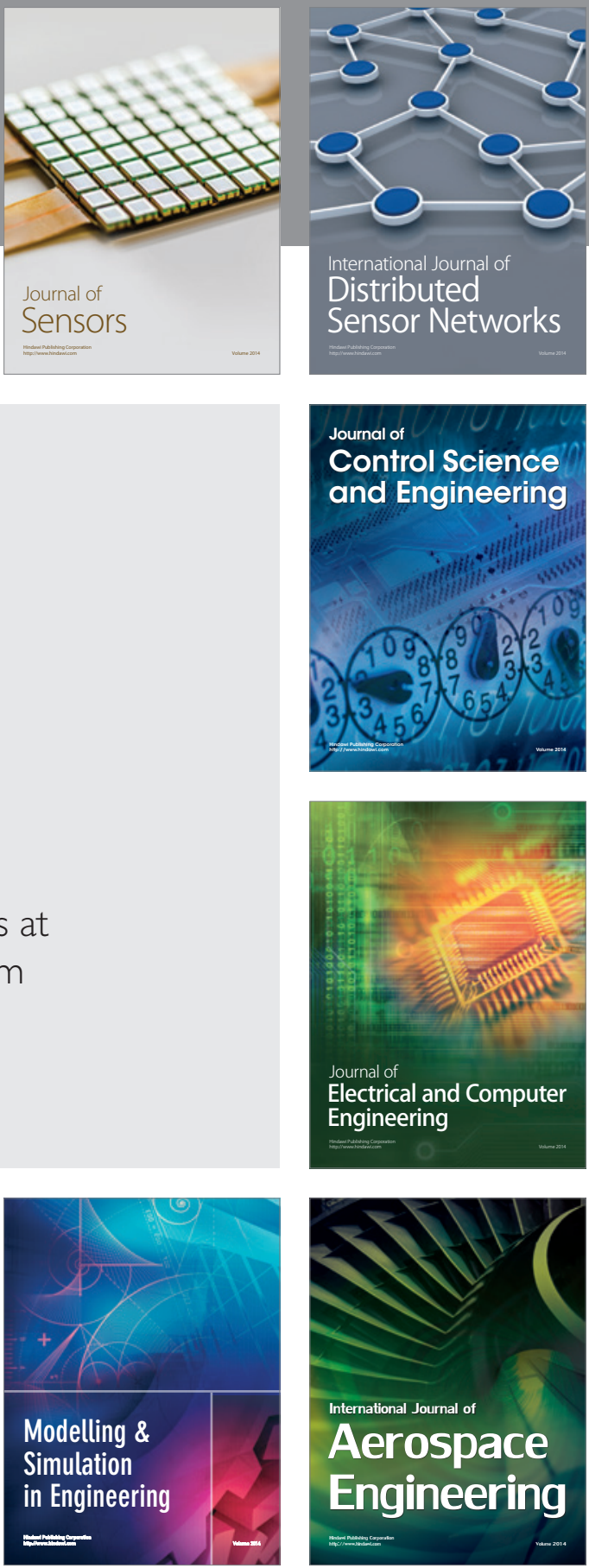

Journal of

Control Science

and Engineering
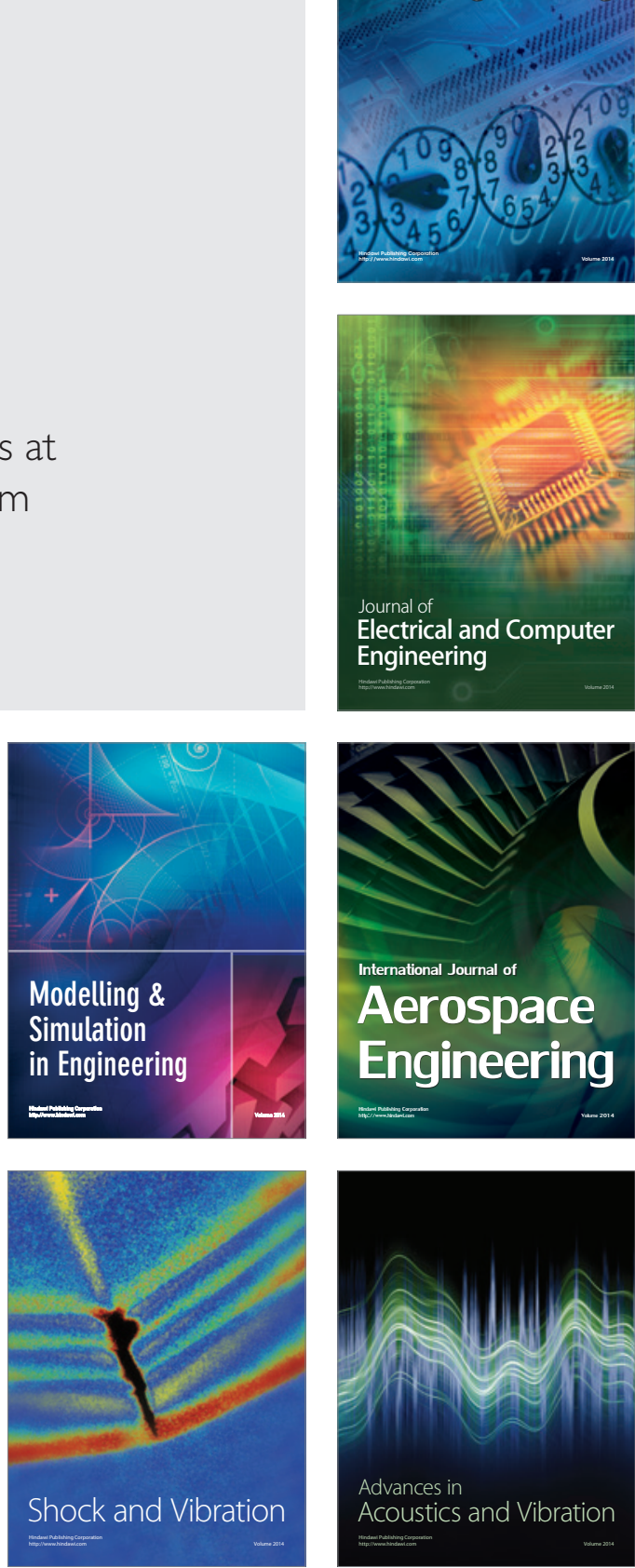